\title{
Complete convergence of randomly weighted END sequences and its application
}

Penghua $\mathrm{Li}^{1}$, Xiaoqin $\mathrm{Li}^{2^{*}}$ and Kehan $\mathrm{Wu}^{3}$

"Correspondence:

lixiaoqin1983@163.com

${ }^{2}$ School of Mathematical Sciences,

Anhui University, Hefei, 230601

China

Full list of author information is

available at the end of the article

\begin{abstract}
We investigate the complete convergence of partial sums of randomly weighted extended negatively dependent (END) random variables. Some results of complete moment convergence, complete convergence and the strong law of large numbers for this dependent structure are obtained. As an application, we study the convergence of the state observers of linear-time-invariant systems. Our results extend the corresponding earlier ones.
\end{abstract}

MSC: 60E15; 60F15

Keywords: complete convergence; randomly weighted; END sequences; strong law of large numbers

\section{Introduction}

Let us recall the concept of extended negatively dependent (END) random variables which was introduced by Liu [1].

Definition 1.1 We call random variables $\left\{X_{n}, n \geq 1\right\}$ to be END if there exists a positive constant $M$ such that both

$$
P\left(X_{i}>x_{i}, i=1,2, \ldots, n\right) \leq M \prod_{i=1}^{n} P\left(X_{i}>x_{i}\right)
$$

and

$$
P\left(X_{i} \leq x_{i}, i=1,2, \ldots, n\right) \leq M \prod_{i=1}^{n} P\left(X_{i} \leq x_{i}\right)
$$

hold for each $n \geq 1$ and all real numbers $x_{1}, x_{2}, \ldots, x_{n}$.

Obviously, for all $1 \leq i \leq n$, let $x_{i}=-\infty$ or $x_{i}=+\infty$ in Definition 1.1, it is easy to see that the dominating coefficient $M \geq 1$. If the dominating coefficient $M$ is 1 , then END random variables reduce to NOD random variables which contain NA random variables and NSD random variables (see Joag-Dev and Proschan [2], Hu [3] and Wang et al. [4]). Various examples of NA random variables and related fields can be found in Bulinski and Shaskin [5], Prakasa Rao [6], Oliveira [7] and the references therein. In view of the importance of

\section{Springer}


END random variables, many researchers pay attention to the study of END. For example, Liu $[1,8]$ studied the precise large deviations and moderate deviations of END sequence with heavy tails; Chen et al. [9] obtained strong law of large numbers of END sequence and gave some applications to the risk theory and renewal theory; Shen [10] obtained some moment inequalities of END sequence; Wang et al. [11] and Hu et al. [12] investigated the complete convergence for END sequences; Wang et al. [13] and Yang et al. [14] investigated the nonparametric regression model under END errors; Yang et al. [15] obtained some large deviation results of nonlinear regression models under END errors; Wang et al. [16] established some exponential inequality for $m$-END sequence; Deng et al. [17] studied the Hajek-Renyi-type inequality and strong law of large numbers for END sequences, etc. Furthermore, there are many works on the negatively dependent random variables. For example, Wang et al. [18] studied the complete convergence for WOD random variables and gave the application to the estimator of nonparametric regression models; Wu et al. [19] obtained some results of complete convergence and complete moment convergence for weighted sums of $m$-NA random variables; Yang and $\mathrm{Hu}[20]$ investigated the complete moment convergence of pairwise NQD random variables; Shen et al. [21] investigated the strong law of large numbers for NOD random variables; Li et al. [22] obtained some results of inverse moment for WOD random variables, etc.

In addition, there are many researchers paying attention to the study of the properties of partial sums of randomly weighted random variables. For example, Thanh and Yin [23] established the complete convergence of partial sums of randomly weighted independent sequences in Banach spaces; Thanh et al. [24] investigated complete convergence of partial sums of randomly weighted $\tilde{\rho}$-mixing sequences; Cabrera et al. [25] and Shen et al. [26] investigated the conditional convergence for partial sums of randomly weighted dependent random variables; Yang et al. [27] and Yao and Lin [28] obtained the complete convergence and moment of maximum normed based on the randomly weighted martingale differences; Han and Xiang [29] obtained the complete moment convergence of double-indexed randomly weighted sums of $\tilde{\rho}$-mixing sequences; Li et al. [30] studied the convergence of partial sums of randomly weighted pairwise NQD sequences, etc.

We aim to investigate the complete convergence of partial sums of randomly weighted END sequences. Some results of complete moment convergence, complete convergence and strong law of large numbers for this dependent structure are obtained. As an application, we study the convergence of state observers of linear-time-invariant systems. We extend some results of Thanh et al. [24], Wang et al. [31] and Yang et al. [32] to the case of randomly weighted END sequences. For the details, see our results in Sections 3 and 4, and the conclusions in Section 5. Some lemmas and proofs of main results are presented in Sections 2 and 6, respectively.

\section{Some lemmas}

Lemma 2.1 (Liu [8]) Let the random variables $\left\{X_{n}, n \geq 1\right\}$ be a sequence of END random variables. If $\left\{f_{n}, n \geq 1\right\}$ is a sequence of all nondecreasing (or nonincreasing) functions, then $\left\{f_{n}\left(X_{n}\right), n \geq 1\right\}$ is also a sequence of END random variables.

Remark 2.1 Let $\left\{X_{n}, n \geq 1\right\}$ be an END sequence and $\left\{Y_{n}, n \geq 1\right\}$ be a sequence of nonnegative and independent random variables, which is independent of $\left\{X_{n}, n \geq 1\right\}$. Let $Z_{n}=X_{n} Y_{n}, n \geq 1$. Combining the definition of END with nonnegative and independent 
of $\left\{Y_{n}\right\}$, we establish, for all real numbers $z_{1}, \ldots, z_{n}$,

$$
\begin{aligned}
P\left(Z_{1} \leq z_{1}, \ldots, Z_{n} \leq z_{n}\right) & =P\left(X_{1} Y_{1} \leq z_{1}, \ldots, X_{n} Y_{n} \leq z_{n}\right) \\
& =\int \cdots \int P\left(X_{1} u_{1} \leq z_{1}, \ldots, X_{n} u_{n} \leq z_{n}\right) d F_{Y_{1}}\left(u_{1}\right) \cdots d F_{Y_{n}}\left(u_{n}\right) \\
& \leq M \int \cdots \int \prod_{i=1}^{n} P\left(X_{i} u_{i} \leq z_{i}\right) d F_{Y_{1}}\left(u_{1}\right) \cdots d F_{Y_{n}}\left(u_{n}\right) \\
& =M \prod_{i=1}^{n} P\left(X_{i} Y_{i} \leq z_{i}\right)=M \prod_{i=1}^{n} P\left(Z_{i} \leq z_{i}\right),
\end{aligned}
$$

by using the fact that $u_{1} X_{1}, u_{2} X_{2}, \ldots, u_{n} X_{n}$ are END random variables following from Lemma 2.1. Similarly, for all real numbers $z_{1}, \ldots, z_{n}$, one has

$$
\begin{aligned}
P\left(Z_{1}>z_{1}, \ldots, Z_{n}>z_{n}\right) & =\int \cdots \int P\left(X_{1} u_{1}>z_{1}, \ldots, X_{n} u_{n}>z_{n}\right) d F_{Y_{1}}\left(u_{1}\right) \cdots d F_{Y_{n}}\left(u_{n}\right) \\
& \leq M \int \cdots \int \prod_{i=1}^{n} P\left(X_{i} u_{i}>z_{i}\right) d F_{Y_{1}}\left(u_{1}\right) \cdots d F_{Y_{n}}\left(u_{n}\right) \\
& =M \prod_{i=1}^{n} P\left(X_{i} Y_{i}>z_{i}\right)=M \prod_{i=1}^{n} P\left(Z_{i}>z_{i}\right) .
\end{aligned}
$$

Therefore, it can be found that $\left\{Z_{n}, n \geq 1\right\}$ is also an END sequence with the same dominating coefficient $M$.

Lemma 2.2 (Shen [10]) Let $p \geq 2$ and $\left\{X_{n}, n \geq 1\right\}$ be an END sequence such that $E X_{n}=0$ and $E\left|X_{n}\right|^{p}<\infty$ for all $n \geq 1$. Then there exists a positive constant $C_{p}$ such that for all $n \geq 1$

$$
E\left|\sum_{i=1}^{n} X_{i}\right|^{p} \leq C_{p}\left\{\sum_{i=1}^{n} E\left|X_{i}\right|^{p}+\left(\sum_{i=1}^{n} E X_{i}^{2}\right)^{p / 2}\right\}
$$

Lemma 2.3 (Sung [33]) Let $\left\{X_{n}, n \geq 1\right\}$ and $\left\{Y_{n}, n \geq 1\right\}$ be sequences of random variables. Then, for any $n \geq 1, q>1, \varepsilon>0$ and $a>0$,

$$
E\left(\left|\sum_{i=1}^{n}\left(X_{i}+Y_{i}\right)\right|-\varepsilon a\right)^{+} \leq\left(\frac{1}{\varepsilon^{q}}+\frac{1}{q-1}\right) \frac{1}{a^{q-1}} E\left|\sum_{i=1}^{n} X_{i}\right|^{q}+E\left|\sum_{i=1}^{n} Y_{i}\right| .
$$

Lemma 2.4 (Adler and Rosalsky [34] and Adler et al. [35]) Let $\left\{X_{n}, n \geq 1\right\}$ be a sequence of random variables which is stochastically dominated by a random variable $X$, i.e. $\sup _{n \geq 1} P\left(\left|X_{n}\right|>t\right) \leq C_{1} P(|X|>t)$ for some positive constant $C_{1}$ and all $t \geq 0$. Then, for all $n \geq 1, \alpha>0$ and $\beta>0$, the following two statements hold:

$$
\begin{aligned}
& E\left[\left|X_{n}\right|^{\alpha} I\left(\left|X_{n}\right| \leq \beta\right)\right] \leq C_{2}\left\{E\left[|X|^{\alpha} I(|X| \leq \beta)\right]+\beta^{\alpha} P(|X|>\beta)\right\}, \\
& E\left[\left|X_{n}\right|^{\alpha} I\left(\left|X_{n}\right|>\beta\right)\right] \leq C_{3} E\left[|X|^{\alpha} I(|X|>\beta)\right] .
\end{aligned}
$$

Consequently, one has $E\left[\left|X_{n}\right|^{\alpha}\right] \leq C_{4} E|X|^{\alpha}$ for all $n \geq 1$. Here $C_{2}, C_{3}, C_{4}$ are some positive constants not depending on $n$. 


\section{The complete convergence for partial sums of randomly weighted END sequences}

In the following, we list two assumptions:

(A.1) Let $\left\{X_{n}, n \geq 1\right\}$ be a mean zero sequence of END random variables stochastically dominated by a random variables $X$.

(A.2) For every $n \geq 1$, let $\left\{A_{n i}, 1 \leq i \leq n\right\}$ be a sequence of independent random variables satisfying that $\left\{A_{n i}, 1 \leq i \leq n\right\}$ is independent of $\left\{X_{n}, n \geq 1\right\}$.

Theorem 3.1 Assume that (A.1) and (A.2) are satisfied. Let $\alpha>1 / 2,1<p<2, E|X|^{p}<\infty$ and $\beta \geq 1$ such that

$$
\sum_{i=1}^{n} E A_{n i}^{2}=O\left(n^{\beta}\right)
$$

Then, for every $\varepsilon>0$,

$$
\sum_{n=1}^{\infty} n^{\alpha p-1-\beta-\alpha} E\left(\left|\sum_{i=1}^{n} A_{n i} X_{i}\right|-\varepsilon n^{\alpha}\right)^{+}<\infty .
$$

So one has

$$
\sum_{n=1}^{\infty} n^{\alpha p-1-\beta} P\left(\left|\sum_{i=1}^{n} A_{n i} X_{i}\right|>\varepsilon n^{\alpha}\right)<\infty .
$$

Theorem 3.2 Assume that (A.1) and (A.2) are satisfied. Let $\alpha>1 / 2, p \geq 2, E|X|^{p}<\infty$ and $\beta \geq 1$ such that

$$
\sum_{i=1}^{n} E\left|A_{n i}\right|^{q}=O\left(n^{\beta}\right)
$$

for some $q>2(\alpha p-1) /(2 \alpha-1)$. Then we also obtain the results of (3.2) and (3.3).

For some $\beta \geq 1$ and $1 / 2<\alpha<(1+\beta) / 2$, we take $\alpha p=1+\beta$ in Theorem 3.2 and establish the following result.

Theorem 3.3 Suppose that (A.1) and (A.2) are fulfilled. Let $\beta \geq 1,1 / 2<\alpha<(1+\beta) / 2$ and $E|X|^{(1+\beta) / \alpha}<\infty$. If

$$
\sum_{i=1}^{n} E\left|A_{n i}\right|^{q}=O\left(n^{\beta}\right) \quad \text { for some } q>\frac{2 \beta}{2 \alpha-1},
$$

then, for every $\varepsilon>0$,

$$
\sum_{n=1}^{\infty} n^{-\alpha} E\left(\left|\sum_{i=1}^{n} A_{n i} X_{i}\right|-\varepsilon n^{\alpha}\right)^{+}<\infty
$$


and

$$
\sum_{n=1}^{\infty} P\left(\left|\sum_{i=1}^{n} A_{n i} X_{i}\right|>\varepsilon n^{\alpha}\right)<\infty .
$$

Thus, by the Borel-Cantelli lemma and (3.7), the strong law of large numbers is as follows:

$$
\frac{1}{n^{\alpha}} \sum_{i=1}^{n} A_{n i} X_{i} \rightarrow 0, \quad \text { a.s. as } n \rightarrow \infty \text {. }
$$

Let $\log x=\ln \max (x, e)$. In addition, for the case $p=1$, we have the following result.

Theorem 3.4 Suppose that (A.1) and (A.2) are fulfilled. Let $\alpha>0$ and $E[|X| \log |X|]<\infty$. If (3.1) holds, then, for every $\varepsilon>0$,

$$
\sum_{n=1}^{\infty} n^{-1-\beta} E\left(\left|\sum_{i=1}^{n} A_{n i} X_{i}\right|-\varepsilon n^{\alpha}\right)^{+}<\infty
$$

So one has

$$
\sum_{n=1}^{\infty} n^{\alpha-1-\beta} P\left(\left|\sum_{i=1}^{n} A_{n i} X_{i}\right|>\varepsilon n^{\alpha}\right)<\infty .
$$

Remark 3.1 If $\beta=1$ in (3.1) and (3.4), then the randomly weighted conditions are

$$
\sum_{i=1}^{n} E A_{n i}^{2}=O(n)
$$

and

$$
\sum_{i=1}^{n} E\left|A_{n i}\right|^{q}=O(n) \quad \text { for some } q>2(\alpha p-1) /(2 \alpha-1)
$$

which are used in Thanh et al. [24]. Under the randomly weighted conditions (3.11), (3.12) and other conditions, Thanh et al. [24] obtained some complete convergence such as

$$
\sum_{n=1}^{\infty} n^{\alpha p-2} P\left(\max _{1 \leq k \leq n}\left|\sum_{i=1}^{k} A_{n i} X_{i}\right|>\varepsilon n^{\alpha}\right)<\infty
$$

for partial sums of randomly weighted $\tilde{\rho}$-mixing sequences. Yang et al. [32] extended the results of Thanh et al. [24] and obtained complete moment convergence such as

$$
\sum_{n=1}^{\infty} n^{\alpha p-2-\alpha} E\left(\max _{1 \leq k \leq n}\left|\sum_{i=1}^{k} a_{n i} X_{i}\right|-\varepsilon n^{\alpha}\right)^{+}<\infty
$$

for partial sums of constant weighted martingale differences. In this paper, we weaken the randomly weighted conditions such as (3.1) and (3.4) for $\beta \geq 1$ and obtain the results of Theorems 3.1-3.4. Generally, we extend the results of Thanh et al. [24] and Yang et al. [32] to the case of randomly weighted END sequences. 


\section{The application to linear-time-invariant systems}

As an application of Theorem 3.3, we study the convergence of the state observers of linear-time-invariant systems in this section.

For $t \geq 0$, we consider a multi-input-single-output (MISO) linear time invariant system as follows:

$$
\left\{\begin{array}{l}
\dot{x}(t)=A x(t)+B u(t), \\
y(t)=C x(t),
\end{array}\right.
$$

where $A \in R^{m_{0} \times m_{0}}, B \in R^{m_{0} \times m_{1}}, C \in R^{1 \times m_{0}}$ are known system matrices, $u(t) \in R^{m_{1}}$ is the control input, $x(t) \in R^{m_{0}}$ is the state and $y(t) \in R$ is the system output. The initial state $x(0)$ is unknown. For some limited observations on $y(t)$, it is interesting to estimate $x(t)$. In the setup, the output $y(t)$ is only measured at a sequence of sampling time instants $\left\{t_{i}\right\}$ with measured values $\gamma\left(t_{i}\right)$ and noised $d_{i}$ such that

$$
y\left(t_{i}\right)=\gamma\left(t_{i}\right)+d_{i}, \quad 1 \leq i \leq n .
$$

We would like to estimate the state $x(t)$ from information on $u(t),\left\{t_{i}\right\}$ and $\left\{\gamma\left(t_{i}\right)\right\}$. Let $G^{\prime}$ denote the transpose of $G$. In order to proceed, we need the following assumption.

Assumption 4.1 The system (4.1) is observable, i.e., the observability matrix

$$
W_{o}^{\prime}=\left[C^{\prime},(C A)^{\prime}, \ldots,\left(C A^{m_{0}-1}\right)^{\prime}\right]
$$

has full rank.

The solution to system (4.1) can be checked:

$$
x(t)=e^{A\left(t-t_{0}\right)} x\left(t_{0}\right)+\int_{t_{0}}^{t} e^{A(t-\tau)} B u(\tau) d \tau .
$$

From $\left\{t_{i}, 1 \leq i \leq n\right\}$, it follows that

$$
\gamma\left(t_{i}\right)+d_{i}=y\left(t_{i}\right)=C e^{A\left(t_{i}-t_{n}\right)} x\left(t_{n}\right)+C \int_{t_{n}}^{t_{i}} e^{A\left(t_{i}-\tau\right)} B u(\tau) d \tau .
$$

Denote

$$
v\left(t_{i}, t_{n}\right)=C \int_{t_{n}}^{t_{i}} e^{A\left(t_{i}-\tau\right)} B u(\tau) d \tau .
$$

So this leads to the observation

$$
C e^{A\left(t_{i}-t_{n}\right)} x\left(t_{n}\right)=\gamma\left(t_{i}\right)-v\left(t_{i}, t_{n}\right)+d_{i}, \quad 1 \leq i \leq n .
$$


Define

$$
\Phi_{n}=\left[\begin{array}{c}
C e^{A\left(t_{1}-t_{n}\right)} \\
\vdots \\
C e^{A\left(t_{n-1}-t_{n}\right)} \\
C
\end{array}\right], \quad \Gamma_{n}=\left[\begin{array}{c}
\gamma\left(t_{1}\right) \\
\vdots \\
\gamma\left(t_{n-1}\right) \\
\gamma\left(t_{n}\right)
\end{array}\right], \quad V_{n}=\left[\begin{array}{c}
v\left(t_{1}, t_{n}\right) \\
\vdots \\
v\left(t_{n-1}, t_{n}\right) \\
0
\end{array}\right], \quad D_{n}=\left[\begin{array}{c}
d_{1} \\
\vdots \\
d_{n-1} \\
d_{n}
\end{array}\right] .
$$

Then we rewrite (4.2) as follows:

$$
\Phi_{n} x\left(t_{n}\right)=\Gamma_{n}-V_{n}+D_{n}
$$

Suppose that $\Phi_{n}$ is full rank, which will be established later. Then the least-squares estimator of $x\left(t_{n}\right)$ is given by

$$
\hat{x}\left(t_{n}\right)=\left(\Phi_{n}^{\prime} \Phi_{n}\right)^{-1} \Phi_{n}^{\prime}\left(\Gamma_{n}-V_{n}\right) .
$$

Combining (4.3) with (4.4), the estimation error for $x\left(t_{n}\right)$ at $t_{n}$ is presented as

$$
e\left(t_{n}\right)=\hat{x}\left(t_{n}\right)-x\left(t_{n}\right)=\left(\Phi_{n}^{\prime} \Phi_{n}\right)^{-1} \Phi_{n}^{\prime} D_{n}=\left(\frac{1}{n^{r}} \Phi_{n}^{\prime} \Phi_{n}\right)^{-1} \frac{1}{n^{r}} \Phi_{n}^{\prime} D_{n} \quad \text { for some } \frac{1}{2}<r<1
$$

In order to obtain the convergence, one must consider a typical entry in $\frac{1}{n^{r}} \Phi_{n}^{\prime} D_{n}$. By the Cayley-Hamilton theorem [36], the matrix exponential can be expressed by a polynomial function of $A$ of order at most $m_{0}-1$ :

$$
e^{A t}=\alpha_{1}(t) I+\cdots+\alpha_{m_{0}}(t) A^{m_{0}-1},
$$

where the time functions $\alpha_{i}(t)$ can be derived by the Lagrange-Hermite interpolation method [36]. So one has

$$
C e^{A\left(t_{i}-t_{n}\right)}=\left[\alpha_{1}\left(t_{i}-t_{n}\right), \ldots, \alpha_{m_{0}}\left(t_{i}-t_{n}\right)\right]\left[\begin{array}{c}
C \\
C A \\
\vdots \\
C A^{m_{0}-1}
\end{array}\right]=\varphi^{\prime}\left(t_{i}-t_{n}\right) W_{o},
$$

where $\varphi^{\prime}\left(t_{i}-t_{n}\right)=\left[\alpha_{1}\left(t_{i}-t_{n}\right), \ldots, \alpha_{m_{0}}\left(t_{i}-t_{n}\right)\right]$ and $W_{o}$ is the observability matrix.

Denote

$$
\Psi_{n}=\left[\begin{array}{c}
\varphi^{\prime}\left(t_{1}-t_{n}\right) \\
\vdots \\
\varphi^{\prime}(0)
\end{array}\right] .
$$

Then

$$
\Phi_{n}=\Psi_{n} W_{o}
$$


which reduces to

$$
\begin{aligned}
& \frac{1}{n^{r}} \Phi_{n}^{\prime} \Phi_{n}=W_{o}^{\prime} \frac{1}{n^{r}} \Psi_{n}^{\prime} \Psi_{n} W_{o}, \\
& \frac{1}{n^{r}} \Phi_{n}^{\prime} D_{n}=\frac{1}{n^{r}} W_{o}^{\prime} \Psi_{n}^{\prime} D_{n} .
\end{aligned}
$$

As a result, one has for any $r>0$

$$
e\left(t_{n}\right)=\left(\frac{1}{n^{r}} \Phi_{n}^{\prime} \Phi_{n}\right)^{-1} \frac{1}{n^{r}} \Phi_{n}^{\prime} D_{n}=W_{o}^{-1}\left(\frac{1}{n^{r}} \Psi_{n}^{\prime} \Psi_{n}\right)^{-1} \frac{1}{n^{r}} \Psi_{n}^{\prime} D_{n} .
$$

By Assumption 4.1, it can be found that $W_{0}^{-1}$ exists. The convergence analysis will be established by the sufficiently conditions: $\frac{1}{n^{r}} \Psi_{n}^{\prime} D_{n} \rightarrow 0$, a.s., and $\frac{1}{n^{r}} \Psi_{n}^{\prime} \Psi_{n} \geq \lambda I$, a.s., for some $\lambda>0$. So we need the following PE condition which was used in Thanh et al. [24] and Wang et al. [31].

Assumption 4.2 For some $1 / 2<r<1$,

$$
\lambda=\inf _{n \geq 1} \sigma_{\min }\left(\frac{1}{n^{r}} \Psi_{n}^{\prime} \Psi_{n}\right)>0, \quad \text { a.s., }
$$

where $\sigma_{\min }(H)$ is the small eigenvalue of $H$ for a suitable symmetric $H$.

We focus on the convergence of partial sums of randomly weighted END random variables such that

$$
\frac{1}{n^{r}} \sum_{i=1}^{n} A_{n i} d_{i}
$$

for some $1 / 2<r<1$. Since a typical entry of $\frac{1}{n^{r}} \Psi_{n}^{\prime} D_{n}$ is

$$
\frac{1}{n^{r}} \sum_{i=1}^{n} \alpha_{j}\left(t_{i}-t_{n}\right) d_{i}
$$

the convergence analysis for $e\left(t_{n}\right)$ is a special case of (4.7). Note that when the sampling time sequence is a random process, so are $\alpha_{j}\left(t_{i}-t_{n}\right)$ in (4.8), rendering a randomly weighted noise driven by END random variables. As an application of Theorem 3.3, we obtain the following theorem.

Theorem 4.1 Let $\beta \geq 1,1 / 2<r<1$ and Assumptions 4.1 and 4.2 hold. Suppose that $\left\{d_{n}, n \geq 1\right\}$ is an END sequences stochastically dominated by a random variable $d$ with $E|d|^{(1+\beta) / r}<\infty$. Suppose that, for some $q>\frac{2 \beta}{2 r-1}$, one has

$$
\sum_{i=1}^{n} E\left|\alpha_{j}\left(t_{i}-t_{n}\right)\right|^{q}=O\left(n^{\beta}\right)
$$

where $1 \leq j \leq m_{0}$. Then

$$
\frac{1}{n^{r}}\left\|\Psi_{n}^{\prime} D_{n}\right\| \rightarrow 0, \quad \text { a.s. }
$$


Consequently,

$$
e\left(t_{n}\right) \rightarrow 0, \quad \text { a.s. }
$$

Remark 4.1 If $\left\{\varphi\left(t_{i}-t_{n}\right)\right\}$ is uniformly bounded, a.s., then the condition (4.9) holds with $\beta=1$ for any $q$. Wang et al. [31] obtained (4.11) for constant weighted $\tilde{\rho}$-mixing errors (see Theorem 4 of Wang et al. [31]). Thanh et al. [24] extended the result of Wang et al. [31] to randomly weighted $\tilde{\rho}$-mixing errors (see Theorem 4.1 of Thanh et al. [24]). Yang et al. [32] obtained the result (4.11) for the case of constant weighted martingale differences (see Theorem 11 of Yang et al. [32]). Generally, our Theorem 4.1 generalize the results of Thanh et al. [24], Wang et al. [31] and Yang et al. [32] to the case of randomly weighted END errors.

\section{Conclusions}

In this paper, we investigate the complete convergence of partial sums of randomly weighted END random variables. Some results of complete moment convergence, complete convergence and strong law of large numbers for this dependent structure are presented (see our Theorems 3.1-3.4). As an application of Theorem 3.3, we study the convergence of the state observers of linear-time-invariant systems and obtain the result of the strong law of large numbers for the systems (see our Theorem 4.1). Therefore, we extend some results of Thanh et al. [24], Wang et al. [31] and Yang et al. [32] to the case of randomly weighted END sequences. Furthermore, END random variables contain NA random variables, NOD random variables and NSD random variables, so the results obtained in this paper hold true for these negatively dependent random variables.

\section{The proofs of main results}

In the proofs, $C, C_{1}, C_{2}, \ldots$ denote some positive constants not depending on $n$.

Proof of Theorem 3.1 Since $A_{n i} X_{i}=A_{n i}^{+} X_{i}-A_{n i}^{-} X_{i}$, without loss of generality, we assume $A_{n i} \geq 0$ in the proof. For $n \geq 1$ and $1 \leq i \leq n$, let

$$
\begin{aligned}
& X_{n i}=-n^{\alpha} I\left(X_{i}<-n^{\alpha}\right)+X_{i} I\left(\left|X_{i}\right| \leq n^{\alpha}\right)+n^{\alpha} I\left(X_{i}>n^{\alpha}\right), \\
& \tilde{X}_{n i}=n^{\alpha} I\left(X_{i}<-n^{\alpha}\right)+X_{i} I\left(\left|X_{i}\right|>n^{\alpha}\right)-n^{\alpha} I\left(X_{i}>n^{\alpha}\right) .
\end{aligned}
$$

It can be found that

$$
A_{n i} X_{i}=\left[A_{n i} X_{n i}-E\left(A_{n i} X_{n i}\right)\right]+E\left(A_{n i} X_{n i}\right)+A_{n i} \tilde{X}_{n i}, \quad 1 \leq i \leq n .
$$

Therefore, by Lemma 2.3 with $a=n^{\alpha}$ and $q=2$, we obtain

$$
\begin{aligned}
& \sum_{n=1}^{\infty} n^{\alpha p-1-\beta-\alpha} E\left(\left|\sum_{i=1}^{n} A_{n i} X_{i}\right|-\varepsilon n^{\alpha}\right)^{+} \\
& \quad \leq C_{1} \sum_{n=1}^{\infty} n^{\alpha p-1-\beta-2 \alpha} E\left|\sum_{i=1}^{n}\left[A_{n i} X_{n i}-E\left(A_{n i} X_{n i}\right)\right]\right|^{2}
\end{aligned}
$$




$$
\begin{aligned}
& \quad+\sum_{n=1}^{\infty} n^{\alpha p-1-\beta-\alpha} E\left|\sum_{i=1}^{n} A_{n i} \tilde{X}_{n i}\right|+\sum_{n=1}^{\infty} n^{\alpha p-1-\beta-\alpha}\left|\sum_{i=1}^{n} E\left(A_{n i} X_{n i}\right)\right| \\
& :=H_{1}+H_{2}+H_{3} .
\end{aligned}
$$

Combining (3.1) with Hölder's inequality, one has

$$
\sum_{i=1}^{n} E\left|A_{n i}\right| \leq\left(\sum_{i=1}^{n} E A_{n i}^{2}\right)^{1 / 2}\left(\sum_{i=1}^{n} 1\right)^{1 / 2} \leq C_{1} n^{\frac{\beta+1}{2}} \leq C_{1} n^{\beta},
$$

by using the fact $\beta \geq 1$.

Since, for every $n \geq 1,\left\{A_{n i}, 1 \leq i \leq n\right\}$ is independent of the sequence $\left\{X_{n}, n \geq 1\right\}$, one has by Markov's inequality, Lemma 2.4, (6.2) and $E|X|^{p}<\infty(p>1)$

$$
\begin{aligned}
H_{2} & \leq 3 \sum_{n=1}^{\infty} n^{\alpha p-1-\beta-\alpha} \sum_{i=1}^{n} E\left|A_{n i}\right| E\left|X_{i}\right| I\left(\left|X_{i}\right|>n^{\alpha}\right) \\
& \leq C_{1} \sum_{n=1}^{\infty} n^{\alpha p-1-\alpha} E\left[|X| \mid I\left(|X|>n^{\alpha}\right)\right] \\
& =C_{1} \sum_{n=1}^{\infty} n^{\alpha p-1-\alpha} \sum_{m=n}^{\infty} E\left[|X| \mid I\left(m<|X|^{1 / \alpha} \leq m+1\right)\right] \\
& =C_{1} \sum_{m=1}^{\infty} E\left[|X| \mid I\left(m<|X|^{1 / \alpha} \leq m+1\right)\right] \sum_{n=1}^{m} n^{\alpha(p-1)-1} \\
& \left.\leq C_{2} \sum_{m=1}^{\infty} m^{\alpha p-\alpha} E|X| I\left(m<|X|^{1 / \alpha} \leq m+1\right)\right] \leq C_{3} E|X|^{p}<\infty .
\end{aligned}
$$

In addition, it can be seen that $E\left(A_{n i} X_{i}\right)=E A_{n i} E X_{i}=0,1 \leq i \leq n, n \geq 1$. So, by the proof of (6.3), we have

$$
\begin{aligned}
H_{3}= & \sum_{n=1}^{\infty} n^{\alpha p-1-\beta-\alpha} \mid \sum_{i=1}^{n}\left[-n^{\alpha} E A_{n i} I\left(X_{i}<-n^{\alpha}\right)-E A_{n i} X_{i} I\left(\left|X_{i}\right|>n^{\alpha}\right) \cdots\right. \\
& \left.+\cdots n^{\alpha} E A_{n i} X_{i} I\left(\left|X_{i}\right|>n^{\alpha}\right)\right] \mid \\
\leq & C_{1} \sum_{n=1}^{\infty} n^{\alpha p-1-\beta-\alpha} \sum_{i=1}^{n} E\left|A_{n i}\right| E\left[\left|X_{i}\right| I\left(\left|X_{i}\right|>n^{\alpha}\right)\right] \leq C_{2} E|X|^{p}<\infty .
\end{aligned}
$$

In view of Lemma 2.1, one sees that $\left\{X_{n i}, 1 \leq i \leq n\right\}$ are END random variables. Combining the assumption of $\left\{A_{n i}\right\}$ with Remark 2.1, we establish that $\left\{\left[A_{n i} X_{n i}-E\left(A_{n i} X_{n i}\right)\right], 1 \leq i \leq\right.$ $n$ ) are mean zero END random variables with the same dominating coefficient. So, by Markov's inequality, (3.1), Lemma 2.2 with $p=2$ and Lemma 2.4, we get

$$
\begin{aligned}
H_{1} & =C_{1} \sum_{n=1}^{\infty} n^{\alpha p-1-\beta-2 \alpha} E\left|\sum_{i=1}^{n}\left[A_{n i} X_{n i}-E\left(A_{n i} X_{n i}\right)\right]\right|^{2} \\
& \leq C_{2} \sum_{n=1}^{\infty} n^{\alpha p-1-\beta-2 \alpha} \sum_{i=1}^{n} E\left(A_{n i} X_{n i}\right)^{2}
\end{aligned}
$$




$$
\begin{aligned}
& \leq C_{3} \sum_{n=1}^{\infty} n^{\alpha p-1-2 \alpha} E\left[X^{2} I\left(|X| \leq n^{\alpha}\right)\right]+C_{4} \sum_{n=1}^{\infty} n^{\alpha p-1} E I\left(|X|>n^{\alpha}\right) \\
& :=C_{3} H_{11}+C_{4} H_{12} .
\end{aligned}
$$

Since $p<2$ and $E X^{p}<\infty$, it can be checked that

$$
\begin{aligned}
H_{11} & =\sum_{n=1}^{\infty} n^{\alpha p-1-2 \alpha} \sum_{i=1}^{n} E\left[X^{2} I\left((i-1)^{\alpha}<|X| \leq i^{\alpha}\right)\right] \\
& =\sum_{i=1}^{\infty} E\left[X^{2} I\left((i-1)^{\alpha}<|X| \leq i^{\alpha}\right)\right] \sum_{n=i}^{\infty} n^{\alpha p-1-2 \alpha} \\
& \left.\leq C_{1} \sum_{i=1}^{\infty} E|X|^{p} X^{2-p} I\left((i-1)^{\alpha}<|X| \leq i^{\alpha}\right)\right] i^{\alpha p-2 \alpha} \leq C_{1} E|X|^{p}<\infty .
\end{aligned}
$$

By the proof of (6.3), it follows that

$$
H_{12} \leq \sum_{n=1}^{\infty} n^{\alpha p-1-\alpha} E\left[|X| I\left(|X|>n^{\alpha}\right)\right] \leq C E|X|^{p}<\infty
$$

Combining (6.1) with (6.3)-(6.7), we can get (3.2) immediately. Moreover, by (3.2) and Remark 2.6 of Sung [33], for every $\varepsilon>0$, it can be argued that

$$
\begin{aligned}
\infty & >\sum_{n=1}^{\infty} n^{\alpha p-1-\beta-\alpha} E\left(\left|\sum_{i=1}^{n} A_{n i} X_{i}\right|-\varepsilon n^{\alpha}\right)^{+} \\
& \geq \sum_{n=1}^{\infty} n^{\alpha p-1-\beta} \int_{0}^{\varepsilon n^{\alpha}} P\left(\left|\sum_{i=1}^{n} A_{n i} X_{i}\right|-\varepsilon n^{\alpha}>t\right) d t \\
& \geq \varepsilon \sum_{n=1}^{\infty} n^{\alpha p-1-\beta} P\left(\left|\sum_{i=1}^{n} A_{n i} X_{i}\right|>2 \varepsilon n^{\alpha}\right),
\end{aligned}
$$

which implies (3.3).

Proof of Theorem 3.2 We use the same notation in the proof of Theorem 3.1. Obviously, by $p \geq 2$, it is easy to see that $q>2(\alpha p-1) /(2 \alpha-1) \geq 2$. Consequently, for any $1 \leq r \leq 2$, by Hölder's inequality and condition (3.4), one has

$$
\sum_{i=1}^{n} E\left|A_{n i}\right|^{r} \leq\left(\sum_{i=1}^{n} E\left|A_{n i}\right|^{q}\right)^{r / q}\left(\sum_{i=1}^{n} 1\right)^{1-r / q} \leq C_{1} n^{\beta \frac{r}{q}+1-\frac{r}{q}}
$$

It can be seen that $\beta \frac{r}{q}+1-\frac{r}{q}-\beta=(\beta-1)\left(\frac{r}{q}-1\right) \leq 0$. So one has $\sum_{i=1}^{n} E\left|A_{n i}\right|=O\left(n^{\beta}\right)$. Together with (6.1), (6.3), (6.4) and (6.9), we obtain $H_{2}<\infty$ and $H_{3}<\infty$. Therefore, we have to prove that $H_{1}<\infty$. Since $q>2$, similar to the proof of (6.5), by Lemma 2.2 , it 
follows that

$$
\begin{aligned}
H_{1}= & C_{1} \sum_{n=1}^{\infty} n^{\alpha p-1-\beta-q \alpha} E\left|\sum_{i=1}^{n}\left[A_{n i} X_{n i}-E\left(A_{n i} X_{n i}\right)\right]\right|^{q} \\
\leq & C_{2} \sum_{n=1}^{\infty} n^{\alpha p-1-\beta-q \alpha}\left(\sum_{i=1}^{n} E\left[A_{n i} X_{n i}-E\left(A_{n i} X_{n i}\right)\right]^{2}\right)^{q / 2} \\
& +C_{2} \sum_{n=1}^{\infty} n^{\alpha p-1-\beta-q \alpha} \sum_{i=1}^{n} E\left|A_{n i} X_{n i}-E\left(A_{n i} X_{n i}\right)\right|^{q} \\
:= & C_{2} H_{11}+C_{2} H_{12} .
\end{aligned}
$$

Obviously, for $1 \leq i \leq n$, by Lemma 2.4 , it follows that

$$
\begin{aligned}
E[ & \left.A_{n i} X_{n i}-E\left(A_{n i} X_{n i}\right)\right]^{2} \\
& \leq C E A_{n i}^{2} E X_{n i}^{2} \\
& \leq C E A_{n i}^{2}\left\{E\left[X^{2} I\left(|X| \leq n^{\alpha}\right)\right]+n^{2 \alpha} P\left(|X|>n^{\alpha}\right)\right\} \\
& \leq C E A_{n i}^{2}\left\{E\left[X^{2} I\left(|X| \leq n^{\alpha}\right)\right]+E\left[X^{2} I\left(|X|>n^{\alpha}\right)\right]\right\}=C E A_{n i}^{2} E X^{2} .
\end{aligned}
$$

By $p \geq 2$ and $E|X|^{p}<\infty$, one concludes that $E X^{2}<\infty$. Thus, we take (6.9) with $r=2$ and (6.11), and establish

$$
\begin{aligned}
H_{11} & \leq C_{3} \sum_{n=1}^{\infty} n^{\alpha p-1-\beta-q \alpha}\left(\sum_{i=1}^{n} E A_{n i}^{2} E X^{2}\right)^{q / 2} \\
& \leq C_{4} \sum_{n=1}^{\infty} n^{\alpha p-1-\beta-q \alpha+(2 \beta / q+1-2 / q) q / 2} \\
& =C_{4} \sum_{n=1}^{\infty} n^{\alpha p-q \alpha+q / 2-2}<\infty,
\end{aligned}
$$

by the fact $q>2(\alpha p-1) /(2 \alpha-1)$. In addition, by the $C_{r}$ inequality, Lemma 2.4 and (3.4),

$$
\begin{aligned}
H_{12} & \leq C_{5} \sum_{n=1}^{\infty} n^{\alpha p-1-\beta-q \alpha} \sum_{i=1}^{n} E\left|A_{n i}\right|^{q} E\left|X_{n i}\right|^{q} \\
& \leq C_{6} \sum_{n=1}^{\infty} n^{\alpha p-1-q \alpha} E\left[|X|^{q} I\left(|X| \leq n^{\alpha}\right)\right]+C_{7} \sum_{n=1}^{\infty} n^{\alpha p-1} P\left(|X|>n^{\alpha}\right) \\
& :=C_{6} H_{12}^{*}+C_{7} H_{12}^{* *} .
\end{aligned}
$$

By $p \geq 2$ and $\alpha>1 / 2$, one has $2(\alpha p-1) /(2 \alpha-1)-p \geq 0$, which implies $q>p$. So, by $E|X|^{p}<$ $\infty$, it can be argued that

$$
\begin{aligned}
H_{12}^{*} & =\sum_{n=1}^{\infty} n^{\alpha p-1-q \alpha} \sum_{i=1}^{n} E\left[|X|^{q} I\left((i-1)^{\alpha}<|X| \leq i^{\alpha}\right)\right] \\
& =\sum_{i=1}^{\infty} E\left[|X|^{q} I\left((i-1)^{\alpha}<|X| \leq i^{\alpha}\right)\right] \sum_{n=i}^{\infty} n^{\alpha p-1-q \alpha}
\end{aligned}
$$




$$
\begin{aligned}
& \leq C_{1} \sum_{i=1}^{\infty} E\left[|X|^{p}|X|^{q-p} I\left((i-1)^{\alpha}<|X| \leq i^{\alpha}\right)\right] i^{\alpha p-q \alpha} \\
& \leq C_{1} E|X|^{p}<\infty .
\end{aligned}
$$

By the proof of (6.3), one has

$$
H_{12}^{* *} \leq \sum_{n=1}^{\infty} n^{\alpha p-1-\alpha} E\left[|X| I\left(|X|>n^{\alpha}\right)\right] \leq C E|X|^{p}<\infty .
$$

Consequently, by (6.10) and (6.12)-(6.15), we obtain $H_{1}<\infty$. So, we obtain the result (3.2). Finally, by the proof of (6.8), (3.3) also holds true.

Proof of Theorem 3.3 For some $\beta \geq 1$ and $\alpha>(1+\beta) / 2$, we take $p=(1+\beta) / \alpha$ and have $\alpha p=1+\beta$. Applying Theorem 3.2, we obtain (3.6) and (3.7) immediately. Combining (3.7) with the Borel-Cantelli lemma, we establish the result of (3.8).

Proof of Theorem 3.4 Similar to the proof of Theorem 3.1, by Lemma 2.3, we can check that

$$
\begin{aligned}
\sum_{n=1}^{\infty} n^{-1-\beta} E\left(\left|\sum_{i=1}^{n} A_{n i} X_{i}\right|-\varepsilon n^{\alpha}\right)^{+} \\
\leq C_{1} \sum_{n=1}^{\infty} n^{-1-\beta-\alpha} E\left|\sum_{i=1}^{n}\left[A_{n i} X_{n i}-E\left(A_{n i} X_{n i}\right)\right]\right|^{2} \\
\quad+\sum_{n=1}^{\infty} n^{-1-\beta} E\left|\sum_{i=1}^{n} A_{n i} \tilde{X}_{n i}\right|+\sum_{n=1}^{\infty} n^{-1-\beta}\left|\sum_{i=1}^{n} E\left(A_{n i} X_{n i}\right)\right| \\
:=Q_{1}+Q_{2}+Q_{3} .
\end{aligned}
$$

By the proof of (6.3), it follows that

$$
\begin{aligned}
Q_{2} & \leq 3 \sum_{n=1}^{\infty} n^{-1} E\left[|X| I\left(|X|>n^{\alpha}\right)\right]=3 \sum_{n=1}^{\infty} n^{-1} \sum_{m=n}^{\infty} E\left[|X| I\left(m<|X|^{1 / \alpha} \leq m+1\right)\right] \\
& =3 \sum_{m=1}^{\infty} E\left[|X| I\left(m<|X|^{1 / \alpha} \leq m+1\right)\right] \sum_{n=1}^{m} n^{-1} \\
& \left.\leq C_{1} \sum_{m=1}^{\infty} \log m E|X| I\left(m<|X|^{1 / \alpha} \leq m+1\right)\right] \leq C_{2} E[|X| \log |X|]<\infty
\end{aligned}
$$

Similarly, by the proof of (6.4), one has

$$
Q_{3} \leq C_{1} \sum_{n=1}^{\infty} n^{-1} E\left[|X| I\left(|X|>n^{\alpha}\right)\right] \leq C_{2} E[|X| \log |X|]<\infty .
$$


Moreover, by the proof of (6.5), we obtain

$$
\begin{aligned}
Q_{1} & \leq C_{1} \sum_{n=1}^{\infty} n^{-1-\beta-\alpha} \sum_{i=1}^{n} E\left(A_{n i} X_{n i}\right)^{2}=C_{1} \sum_{n=1}^{\infty} n^{-1-\beta-\alpha} \sum_{i=1}^{n} E A_{n i}^{2} E X_{n i}^{2} \\
& \leq C_{2} \sum_{n=1}^{\infty} n^{-1-\alpha} E\left[X^{2} I\left(|X| \leq n^{\alpha}\right)\right]+C_{3} \sum_{n=1}^{\infty} n^{\alpha-1} P\left(|X|>n^{\alpha}\right) \\
& \leq C_{2} \sum_{i=1}^{\infty} E\left[X^{2} I\left((i-1)^{\alpha}<|X| \leq i^{\alpha}\right)\right] \sum_{n=i}^{\infty} n^{-1-\alpha}+C_{4} E[|X| \log |X|] \\
& \leq C_{5} \sum_{i=1}^{\infty} E\left[X^{2} I\left((i-1)^{\alpha}<|X| \leq i^{\alpha}\right)\right] i^{-\alpha}+C_{4} E[|X| \log |X|] \\
& \leq C_{6} E|X|+C_{5} E[|X| \log |X|]<\infty .
\end{aligned}
$$

So, by (6.16)-(6.19), (3.9) holds. In addition, by (3.3) with $p=1,(3.10)$ also holds true under the conditions of Theorem 3.4.

Proof of Theorem 4.1 It is easy to check that

$$
\frac{1}{n^{r}} \Psi_{n}^{\prime} D_{n}=\left[\begin{array}{c}
\frac{1}{n^{r}} \sum_{i=1}^{n} \alpha_{1}\left(t_{i}-t_{n}\right) d_{i} \\
\vdots \\
\frac{1}{n^{r}} \sum_{i=1}^{n} \alpha_{m_{0}}\left(t_{i}-t_{n}\right) d_{i}
\end{array}\right]
$$

In order to prove (4.10), it suffices to look at the $j$ th component $\frac{1}{n^{r}} \sum_{i=1}^{n} \alpha_{j}\left(t_{i}-t_{n}\right) d_{i}$ of $\frac{1}{n^{r}} \Psi_{n}^{\prime} D_{n}$. For some $q>\frac{2 \beta}{2 r-1}$, by (4.9), we apply Theorem 3.3 with $\alpha=r, A_{n i}=\alpha_{j}\left(t_{i}-t_{n}\right)$ in (4.8) and $X_{n}=d_{n}$, and obtain the result of (4.10).

Moreover, by Assumption 4.1, $W_{0}^{-1}$ exists. In addition, by (4.6) in Assumption 4.2, $\left(\frac{1}{n^{r}} \Psi_{n}^{\prime} \Psi_{n}\right)^{-1}$ exists and

$$
\sigma_{\max }\left(\left(\frac{1}{n^{r}} \Psi_{n}^{\prime} \Psi_{n}\right)^{-1}\right) \leq \frac{1}{\lambda}, \quad \text { a.s. }
$$

where $\sigma_{\max }(\cdot)$ is the largest eigenvalue. Therefore, combining

$$
e\left(t_{n}\right)=W_{o}^{-1}\left(\frac{1}{n^{r}} \Psi_{n}^{\prime} \Psi_{n}\right)^{-1} \frac{1}{n^{r}} \Psi_{n}^{\prime} D_{n}
$$

with (4.10), we obtain (4.11) immediately.

\section{Acknowledgements}

The authors are deeply grateful to the editors and anonymous referees, whose insightful comments and suggestions have contributed substantially to the improvement of this paper. This work is supported by National Natural Science Foundation of China (11501005, 61403053, 61403115), Natural Science Foundation of Anhui Province (1508085J06, 1608085QA02) and Science Research Project of Anhui Colleges (KJ2017A027, KJ2014A020, KJ2015A065, KJ2016A027)

\section{Competing interests}

The authors declare that they have no competing interests. 


\section{Author details}

'Automotive Electronics Engineering Research Center, College of Automation, Chongqing University of Posts and Telecommunications, Chongqing, 400065, China. ${ }^{2}$ School of Mathematical Sciences, Anhui University, Hefei, 230601, China. ${ }^{3}$ Training Center of Anhui Provincial Electric Power Company, Hefei, 230000, China.

\section{Publisher's Note}

Springer Nature remains neutral with regard to jurisdictional claims in published maps and institutional affiliations.

Received: 5 February 2017 Accepted: 19 July 2017 Published online: 07 August 2017

\section{References}

1. Liu, L: Precise large deviations for dependent random variables with heavy tails. Stat. Probab. Lett. 79, 1290-1298 (2009)

2. Joag-Dev, K, Proschan, F: Negative association of random variables with applications. Ann. Stat. 11, $286-295$ (1983)

3. Hu, TZ: Negatively superadditive dependence of random variables with applications. Chinese J. Appl. Probab. Statist. $16,133-144(2000)$

4. Wang, XJ, Shen, AT, Chen, ZY, Hu, SH: Complete convergence for weighted sums of NSD random variables and its application in the EV regression model. Test 24, 166-184 (2015)

5. Bulinski, AV, Shaskin, A: Limit Theorems for Associated Random Fields and Related Systems. World Scientific, Singapore (2007)

6. Prakasa Rao, BLS: Associated Sequences, Demimartingales and Nonparametric Inference. Springer, Basel (2012)

7. Oliveira, PE: Asymptotics for Associated Random Variables. Springer, Berlin (2012)

8. Liu, L: Necessary and sufficient conditions for moderate deviations of dependent random variables with heavy tails. Sci. China Ser. A 53, 1421-1434 (2010)

9. Chen, $\mathrm{YQ}, \mathrm{Chen}, \mathrm{AY}, \mathrm{Ng}, \mathrm{KW}$ : The strong law of large numbers for extended negatively dependent random variables. J. Appl. Probab. 47, 908-922 (2010)

10. Shen, AT: Probability inequalities for END sequence and their applications. J. Inequal. Appl. 2011, 98 (2011)

11. Wang, XJ, Hu, T-C, Volodin, A, Hu, SH: Complete convergence for weighted sums and arrays of rowwise extended negatively dependent random variables. Commun. Stat., Theory Methods 42, 2391-2401 (2013)

12. Hu, T-C, Rosalsky, A, Wang, KL: Complete convergence theorems for extended negatively dependent random variables. Sankhya, Ser. A 77, 1-29 (2015)

13. Wang, XJ, Zheng, LL, Xu, C, Hu, SH: Complete consistency for the estimator of nonparametric regression models based on extended negatively dependent errors. Statistics 49, 396-407 (2015)

14. Yang, WZ, Xu, HY, Chen, L, Hu, SH: Complete consistency of estimators for regression models based on extended negatively dependent errors. Stat. Pap. (2016, in press). doi:10.1007/s00362-016-0771-X

15. Yang, WZ, Zhao, ZR, Wang, $\mathrm{XH}, \mathrm{Hu}, \mathrm{SH}$ : The large deviation results for the nonlinear regression model with dependent errors. Test 26(2), 261-283 (2017)

16. Wang, $\mathrm{XJ}, \mathrm{Wu}, \mathrm{Y}, \mathrm{Hu}, \mathrm{SH}$ : Exponential probability inequality for $\mathrm{m}$-END random variables and its applications. Metrika 79, 127-147 (2016)

17. Deng, X, Wang, XJ, Xia, FX: Hajek-Renyi-type inequality and strong law of large numbers for END sequences. Commun. Stat., Theory Methods 46(2), 672-682 (2017)

18. Wang, XJ, Xu, C, Hu, T-C, Volodin, A: On complete convergence for widely orthant-dependent random variables and its applications in nonparametric regression models. Test 23(3), 607-629 (2014)

19. $\mathrm{Wu}, \mathrm{YF}, \mathrm{Hu}, \mathrm{T}-\mathrm{C}$ : Complete convergence and complete moment convergence for weighted sums of m-NA random variables. J. Inequal. Appl. 2015, 200 (2015)

20. Yang, WZ: Complete moment convergence of pairwise NQD random variables. Stochastics 87(2), $199-208$ (2015)

21. Shen, AT, Zhang, Y: On the rate of convergence in the strong law of large numbers for negatively orthant-dependent random variables. Commun. Stat., Theory Methods 45(21), 6209-6222 (2016)

22. Li, XQ, Liu, X, Yang, WZ: The inverse moment for widely orthant dependent random variables. J. Inequal. Appl. 2016, $161(2016)$

23. Thanh, LV, Yin, G: Almost sure and complete convergence of randomly weighted sums of independent random elements in Banach spaces. Taiwan. J. Math. 15, 1759-1781 (2011)

24. Thanh, LV, Yin, G, Wang, LY: State observers with random sampling times and convergence analysis of double-indexed and randomly weighted sums of mixing processes. SIAM J. Control Optim. 49, 106-124 (2011)

25. Cabrera, MO, Rosalsky, A, Volodin, A: Some theorems on conditional mean convergence and conditional almost sure convergence for randomly weighted sums of dependent random variables. Test 21, 369-385 (2012)

26. Shen, AT, Wu, RC, Chen, Y, Zhou, Y: Conditional convergence for randomly weighted sums of random variables based on conditional residual $h$-integrability. J. Inequal. Appl. 2013, 122 (2013)

27. Yang, WZ, Wang, YW, Wang, XH, Hu, SH: Complete moment convergence for randomly weighted sums of martingale differences. J. Inequal. Appl. 2013, 396 (2013)

28. Yao, M, Lin, L: The moment of maximum normed randomly weighted sums of martingale differences. J. Inequal. Appl. 2015, 264 (2015)

29. Han, J: Complete moment convergence of double-indexed randomly weighted sums of mixing sequences. J. Inequal. Appl. 2016, 313 (2016)

30. Li, XQ, Zhao, ZR, Yang, WZ, Hu, SH: The inequalities of randomly weighted sums of pairwise NQD sequences and its application to limit theory. J. Math. Inequal. 11, 323-334 (2017)

31. Wang, LY, Li, C, Yin, G, Guo, L, Xu, CZ: State observability and observers of linear-time-invariant systems under irregular sampling and sensor limitations. IEEE Trans. Autom. Control 56, 2639-2654 (2011)

32. Yang, WZ, Wang, XH, Li, XQ, Hu, SH: The convergence of double-indexed weighted sums of martingale differences and its application. Abstr. Appl. Anal. 2014, Article ID 893906 (2014)

33. Sung, SH: Moment inequalities and complete moment convergence. J. Inequal. Appl. 2009, Article ID 271265 (2009) 
34. Adler, A, Rosalsky, A: Some general strong laws for weighted sums of stochastically dominated random variables. Stoch. Anal. Appl. 5, 1-16 (1987)

35. Adler, A, Rosalsky, A, Taylor, RL: Strong laws of large numbers for weighted sums of random elements in normed linear spaces. Int. J. Math. Math. Sci. 12, 507-530 (1989)

36. Ogata, K: Modern Control Engineering, 4th edn. Prentice Hall, Englewood Cliffs (2002)

Submit your manuscript to a SpringerOpen ${ }^{\circ}$ journal and benefit from:

- Convenient online submission

$\checkmark$ Rigorous peer review

Open access: articles freely available online

- High visibility within the field

- Retaining the copyright to your article

Submit your next manuscript at $\gg$ springeropen.com 